\title{
Existence and uniqueness of solution to nonlinear second-order distributional differential equations
}

\author{
Feng Chen*1 (D), Guoju Ye ${ }^{1}$ (D), Wei Liu ${ }^{1}$ (D), Dafang Zhao ${ }^{2}$ (D) \\ ${ }^{1}$ College of Science, Hohai University, Nanjing 210098, P.R. China \\ ${ }^{2}$ School of Mathematics and Statistics, Hubei Normal University, Huangshi, Hubei 435002, P. R. China.
}

\begin{abstract}
The aim of this paper is to obtain solutions in terms of regulated functions to secondorder distributional differential equations for Dirichlet problem. Existence and uniqueness theorems are established by using Schaefer's fixed point theorem and Banach's contraction mapping principle. Examples are given to demonstrate that the results are nontrivial.
\end{abstract}

Keywords. regulated functions, functions of bounded variation, distributional differential equation, Schaefer's fixed point theorem, Banach's contraction mapping principle

Mathematics Subject Classification (2010). 74H20, 81Q15

\section{Introduction}

Differential equations in the following form are considered as measure differential equations (MDEs, for short),

$$
D x=f(t, x)+g(t, x) D u,
$$

in which $D x$ and $D u$ are the distributional derivatives of function $x$ and $u$ of bounded variation in the sense of L. Schwartz distribution, respectively. As a branch of generalized ordinary differential equations (GODEs, for short), MDEs mainly describe the discontinuous solutions caused by the impulsive behavior of the differential system. MDEs serve as good models for a lot of natural phenomena, such as physical process, automatic control problems and biological neural nets (see $[2,7]$ ). Therefore, there has been much attention paid to MDEs. Monteiro and Slavík [9] obtained the existence of the least and greatest solutions to MDEs. In 2017, by using correspondence between MDEs and GODEs, Federson et al. [3] studied the boundedness results for MDEs. For more details, readers are referred to $[8,10,11]$. However, the second order situation

$$
-D^{2} x=f(t, x)+g(t, x) D u,
$$

does not draw enough attention. Moreover, the results in the case when $u$ is a regulated function are few. Here (1.2) is considered as distributional differential equation (DDE, for short) when $u$ is a regulated function.

\footnotetext{
*Corresponding Author.

Email addresses: fenggedii@163.com (F. Chen), yegj@hhu.edu.cn (G. Ye), liuw626@hhu.edu.cn (W. Liu), dafangzhao@163.com (D. Zhao)

Received: 02.03.2018; Accepted: 15.10.2018
} 
In this paper, we wish to investigate the second order distributional differential equation (1.2), $t \in[0,1]$, with the Dirichlet boundary condition

$$
x(0)=x(1)=0,
$$

here in (1.2) $D^{2} x$ stands for the second order distributional derivative of $x$, and $x, u$ : $R \rightarrow R$ are regulated functions. As for the functions $f, g$, they will be introduced later.

The Henstock-Kurzweil-Stieltjes integral, which includes the Lebesgue-Stieltjes integral and the Riemann-Stieltjes integral, is a vital tool for our investigation. Moreover, it is known that the Lebesgue-Stieltjes integral does not include the integration with respect to regulated functions while the Henstock-Kurzweil-Stieltjes integral does. Compared with the Riemann-Stieltjes integral, the Henstock-Kurzweil-Stieltjes integral is more useful. For example, $(H K S) \int_{a}^{b} f d g$ exists if $(R S) \int_{a}^{b} f d g$ exists. However, when $(H K S) \int_{a}^{b} f d g$ exists, $(R S) \int_{a}^{b} f d g$ does not exist if $f$ and $g$ have a common point of discontinuity in the compact interval $[a, b]$. Here "(HKS) $\int$ " stands for the Henstock-Kurzweil-Stieltjes integral and "(RS) $\int$ " stands for the Riemann-Stieltjes integral.

The structure of this paper is as follows. Section 2 is dedicated to some basic notions which will be used later. In Section 3, we restrict our attention to the existence of solutions to the problem (1.2) and (1.3) under certain assumptions, and relative lemmas which are applied to obtain the existence result are also included. Besides, the uniqueness of solution to problem (1.2) and (1.3) is investigated with Banach's contraction mapping principle in Section 4 . We give an example for each result.

\section{Basic notions}

In this section, we review the basic definitions of the Henstock-Kurzweil-Stieltjes integral, functions of bounded variation and regulated functions, which help readers understand the following work.

$\mathfrak{D}=\left\{t_{0}, t_{1}, t_{2}, \ldots, t_{m}\right\}$ is said to be a partition of a closed interval $[a, b]$ if $a=t_{0}<$ $t_{1}<t_{2}<\ldots<t_{m}=b$. The set of partitions of $[a, b]$ is denoted by $\mathfrak{D}[a, b]$. Besides, we call a pair $\left(\tau_{i},\left[t_{i-1}, t_{i}\right]\right)$ to be a tagged interval, in which $\tau_{i} \in\left[t_{i-1}, t_{i}\right]$ is a tag of $\left[t_{i-1}, t_{i}\right]$. A function $\delta:[a, b] \rightarrow(0,+\infty)$ is called a gauge on $[a, b]$. A tagged partition of the interval $[a, b]$ with division points $a=\tau_{0}=t_{0}<t_{1}<t_{2}<\ldots<t_{m}=\tau_{m-1}=b$ and tags $\tau_{i} \in\left[t_{i-1}, t_{i}\right],(i=1,2,3, \ldots, m)$ is called $\delta$ - fine if $\left[t_{i-1}, t_{i}\right] \subseteq\left(\tau_{i}-\delta\left(\tau_{i}\right), \tau_{i}+\delta\left(\tau_{i}\right)\right),(i=$ $1,2,3, \ldots, m)$. Moreover, $\tau_{0}=a, \tau_{m-1}=b$. The set of $\delta$-fine partitions of $[a, b]$ is denoted by $\mathfrak{D}_{\delta}[a, b]$.

With the introduction of $\delta$-fine partitions, we are able to introduce the HenstockKurzweil-Stieltjes integral.

Let $f, g:[a, b] \rightarrow R$. If there exists $J \in R$ for every $\varepsilon>0$, there exists a positive function $\delta>0$, such that for all $\delta$-fine partitions of $\mathfrak{D}_{\delta}[a, b]$,

or

$$
\left|J-\sum_{i=1}^{m-1} f\left(\tau_{i}\right)\left(g\left(t_{i}\right)-g\left(t_{i-1}\right)\right)\right| \leq \varepsilon,
$$

$$
\left|J-\left(f(b) g(b)-f(a) g(a)-\sum_{i=1}^{m} g\left(t_{i-1}\right)\left(f\left(\tau_{i}\right)-f\left(\tau_{i-1}\right)\right)\right)\right| \leq \varepsilon,
$$

then $J$ is called the Henstock-Kurzweil-Stieltjes integral of $f$ on $[a, b]$ with respect to $g$ and denoted by

$$
J=(H K S) \int_{a}^{b} f(t) d g(t)=(H K S) \int_{a}^{b} f d g .
$$

We say that the functions $f$ are called HKS-integrable with respect to $g$. For more details, we refer readers to [14]. 
Remark 2.1. The Henstock-Kurzweil integral, which belongs to the distributional Henstock-Kurzweil integral (see [13]), is a special case of the Henstock-Kurzweil-Stieltjes integral if $g$ is the identity function, then the functions $f$ are said to be HK-integrable. The integral sign will always denote the Henstock-Kurzweil integral or the Henstock-KurzweilStieltjes integral in what follows.

For a given function $f:[a, b] \rightarrow R$ and a given $\mathfrak{D} \in \mathfrak{D}[a, b]$, we denote the variation by

$$
\operatorname{Var}(f, \mathfrak{D}):=\sum_{j=1}^{m}\left|f\left(t_{i}\right)-f\left(t_{i-1}\right)\right| \text {. }
$$

And the total variation of $f$ is denoted by

$$
\operatorname{Var}_{[a, b]} f:=\sup \{\operatorname{Var}(f, \mathfrak{D}) ; \mathfrak{D} \in \mathfrak{D}[a, b]\} .
$$

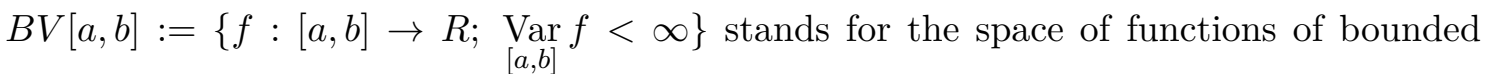

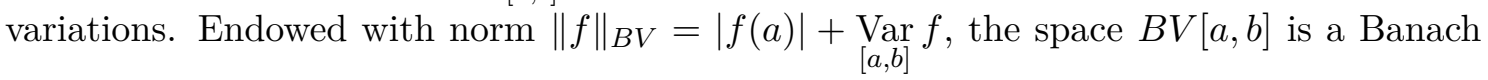
space (see [1]).

Let $R$ be the real line and $[a, b]$ be a closed interval of $R$. A function $f:[a, b] \rightarrow R$ is called a regulated function on $[a, b]$ if the function possesses finite limits

$$
\lim _{s \rightarrow t-} f(s)=f(t-), \quad t \in(a, b] \quad \text { and } \quad \lim _{s \rightarrow t+} f(s)=f(t+), \quad t \in[a, b) .
$$

The space of regulated functions $f:[a, b] \rightarrow R$ is denoted by $G[a, b]$. Endowed with the sup norm $\|f\|=\sup _{t \in[a, b]}|f(t)|$, the space $G[a, b]$ is a Banach space (see [4]). The symbol of norm here is different from the norm in $B V[a, b]$ mentioned above.

Similar to equicontinuous sets in the space of continuous functions, a set $\mathcal{A} \subset G[a, b]$ is called equiregulated if for every $\varepsilon>0$ and $t_{0} \in[a, b]$, there is a $\delta>0$ such that for every $x \in \mathcal{A}$ and $t \in[a, b]$, if $t_{0}-\delta<t<t_{0}$, then $\left|x\left(t_{0}-\right)-x(t)\right|<\varepsilon$ and if $t_{0}<t<t_{0}+\delta$, then $\left|x(t)-x\left(t_{0}+\right)\right|<\varepsilon$.

\section{Existence of solutions to problem (1.2) with the Dirichlet boundary condition (1.3)}

In this part, we present the existence result for problem (1.2) with the Dirichlet boundary condition (1.3), whose proof is based on Schaefer's fixed point theorem, we state as follows.

Lemma 3.1 ([15]). Let $E$ be a normed linear space and $\Phi: E \rightarrow E$ be a compact operator. Suppose that the set

$$
S=\{x \in E \mid x=\lambda \Phi x, \text { for some } \lambda \in(0,1)\}
$$

is bounded. Then $\Phi$ has a fixed point in $E$.

The following several lemmas are vital when we use Schaefer's fixed point theorem to obtain the existence of solutions to problem (1.2) with the Dirichlet boundary condition (1.3).

Lemma $3.2([4])$. A set $\mathcal{A} \subset G[a, b]$ is relatively compact if and only if it is equiregulated and for every $t \in[a, b]$, the set $\{x(t): x \in \mathcal{A}\}$ is bounded.

Lemma $3.3([12])$. Let $f \in B V[a, b]$ and $g \in G[a, b]$, then the integral $\int_{a}^{b} f d g$ exists and the inequality

is true.

$$
\left|\int_{a}^{b} f d g\right| \leq\left(|f(a)|+|f(b)|+\operatorname{Var}_{[a, b]} f\right)\|g\|
$$


Lemma 3.4 ([1]). Let function $g, h \in B V[a, b]$, we have

(a) $g h \in B V[a, b]$;

(b) $\underset{[a, b]}{\operatorname{Var}}(g h) \leq\|f\| \underset{[a, b]}{\operatorname{Var}} g+\|g\| \underset{[a, b]}{\operatorname{Var}} f$;

(c) $\|g\| \leq\|g\|_{B V}$.

Lemma 3.5 ([12]). Let $g \in G[a, b]$ and let $h, h_{n}:[a, b] \rightarrow R$ be such that

$$
\int_{a}^{b} h_{n} d g \text { exists for any } n \in \mathbb{N} \text { and } \lim _{n \rightarrow \infty}\left\|h-h_{n}\right\|_{B V}=0 .
$$

Then $\int_{a}^{b} h d g$ exists and $\lim _{n \rightarrow \infty} \int_{a}^{b} h_{n} d g=\int_{a}^{b} h d g$.

Lemma 3.6 ([6]). If the conditions

(1) $f_{n}(t)(n=1,2, \ldots)$ is HK-integrable and $f_{n}(t) \rightarrow f(t)$ a.e., $t \in[a, b]$,

(2) $g, h$ are HK-integrable and for every $t, g(t) \leq f_{n}(t) \leq h(t)$ a.e., $t \in[a, b]$, are satisfied, then $f(t)$ is HK-integrable and

$$
\lim _{n \rightarrow \infty} \int_{a}^{b} f_{n}(t) d t=\int_{a}^{b} f(t) d t
$$

Here we give the definition of a ball $B_{r}$,

$$
B_{r}=\{x \in G[0,1] \mid\|x\| \leq r\}, \quad r>0 .
$$

Next we impose the following assumptions on $f$ and $g$.

$\left(H_{1}\right) f(\cdot, x(\cdot))$ is HK-integrable for every $x \in G[0,1]$;

$\left(H_{2}\right) f(t, \cdot)$ and $g(t, \cdot)$ are both continuous for every $t \in[0,1]$;

$\left(H_{3}\right)$ There exist HK-integrable functions $f_{1}(\cdot)$ and $f_{2}(\cdot)$ such that $f_{1}(\cdot) \leq f(\cdot, x(\cdot)) \leq$ $f_{2}(\cdot)$ for every $x \in G[0,1]$;

$\left(H_{4}\right) g(\cdot, x(\cdot)) \in B V[0,1],\|g(\cdot, x)\|_{B V} \leq M$, for every $x \in G[0,1], M>0$.

Before our existence results, one lemma concerning the equivalence of integral equations and distributional differential equations is essential.

Lemma 3.7. $x$ is the solution of problem (1.2) with the Dirichlet boundary condition (1.3) if and only if $x$ satisfies the following integral equation

$$
x(t)=\int_{0}^{1} k(t, s) f(s, x(s)) d s+\int_{0}^{1} k(t, s) g(s, x(s)) d u(s),
$$

in which

$$
k(t, s)= \begin{cases}t(1-s), & 0 \leq t \leq s \leq 1 \\ s(1-t), & 0 \leq s \leq t \leq 1\end{cases}
$$

Proof. First, we assume that $x$ is the solution of problem (1.2) with the Dirichlet boundary condition (1.3). Afterwards, by integrating both sides of the equation (1.2) from 0 to $t$ twice, we obtain

$$
x(t)=x(0)+D x(0) t-\left(\int_{0}^{t} \int_{0}^{\tau} f(s, x(s)) d s d \tau+\int_{0}^{t} \int_{0}^{\tau} g(s, x(s)) d u(s) d \tau\right) .
$$

It follows from equation (3.2) with $t=1$ and $x(0)=x(1)=0$ that

$$
D x(0)=\int_{0}^{1} \int_{0}^{\tau} f(s, x(s)) d s d \tau+\int_{0}^{1} \int_{0}^{\tau} g(s, x(s)) d u(s) d \tau .
$$


Inserting (3.3) into (3.2), we get

$$
\begin{aligned}
x(t)= & t \int_{0}^{1} \int_{0}^{\tau} f(s, x(s)) d s d \tau+t \int_{0}^{1} \int_{0}^{\tau} g(s, x(s)) d u(s) d \tau-\int_{0}^{t} \int_{0}^{\tau} f(s, x(s)) d s d \tau \\
& -\int_{0}^{t} \int_{0}^{\tau} g(s, x(s)) d u(s) d \tau \\
= & \int_{0}^{t} s(1-t) f(s, x(s)) d s+\int_{0}^{t} s(1-t) g(s, x(s)) d u(s)+\int_{t}^{1} t(1-s) f(s, x(s)) d s \\
& +\int_{t}^{1} t(1-s) g(s, x(s)) d u(s) \\
= & \int_{0}^{1} k(t, s) f(s, x(s)) d s+\int_{0}^{1} k(t, s) g(s, x(s)) d u(s) .
\end{aligned}
$$

Note that for $t \in[0,1], k(t, \cdot) \in B V[0,1]$, and also $x(1)=x(0)=0$.

Conversely, the second order distributional derivative of both sides of the equation (3.1) is equation (1.2).

The proof is completed.

Remark 3.8. When integrating the right side of the equation (1.2), we cannot apply the Lebesgue-Stieltjes integral since $u$ is a regulated function. Besides, we are not sure if $g$ and $u$ have any common point of discontinuity, thus the Riemann-Stieltjes integral is not applicable. Therefore, the application of the Henstock-Kurzweil-Stieltjes integral is necessary.

The following theorem presents the existence result.

Theorem 3.9. Assume that the assumptions $\left(H_{1}\right),\left(H_{2}\right),\left(H_{3}\right)$ and $\left(H_{4}\right)$ are satisfied. Then problem (1.2) with the Dirichlet boundary condition (1.3) has at least one solution in $G[0,1]$.

Proof. Define an operator $\mathbb{A}$ as follows,

$$
\mathbb{A} x(t)=\int_{0}^{1} k(t, s) f(s, x(s)) d s+\int_{0}^{1} k(t, s) g(s, x(s)) d u(s), \quad t \in[0,1] .
$$

We denote the set $\{x \in G[0,1] \mid x=\lambda \mathbb{A} x$, for some $\lambda \in(0,1)\}$ by $S$.

It follows from Lemma 3.7 that the fixed point of $\mathbb{A}$ is a solution to problem (1.2) with the Dirichlet boundary condition (1.3). We divide the process of proving that the operator $\mathbb{A}$ has a fixed point into four steps.

Step 1. There is $r>0$ such that $\mathbb{A}\left(B_{r}\right) \subseteq B_{r}$.

For every $x \in G[0,1]$ and for every $t \in[0,1]$, under the assumption $\left(H_{3}\right)$ and in virtue of Lemma 3.3, one has

$$
\begin{aligned}
|\mathbb{A} x(t)| & =\left|\int_{0}^{1} k(t, s) f(s, x(s)) d s+\int_{0}^{1} k(t, s) g(s, x(s)) d u(s)\right| \\
& \leq\left|\int_{0}^{1} k(t, s) f_{1}(s, x(s)) d s\right|+\left|\int_{0}^{1} k(t, s) f_{2}(s, x(s)) d s\right|+\underset{[0,1]}{\operatorname{Var}}(k(t, s) g(s, x(s)))\|u\| .
\end{aligned}
$$

Let

$$
\max _{t \in[0,1]}\left(\left|\int_{0}^{1} k(t, s) f_{1}(s) d s\right|+\left|\int_{0}^{1} k(t, s) f_{2}(s) d s\right|\right)=M_{1} .
$$

As a consequence of Lemma 3.4 and the assumption $\left(H_{4}\right)$, for every $t \in[0,1]$ and for every $x \in G[0,1]$,

$$
\underset{s \in[0,1]}{\operatorname{Var}}(k(t, s) g(s, x(s))) \leq\|k(t, \cdot)\| \underset{s \in[0,1]}{\operatorname{Var}} g(s, x(s))+\|g(\cdot, x)\| \underset{s \in[0,1]}{\operatorname{Var}} k(t, s) \leq \frac{3}{2} M,
$$

in which $\underset{s \in[0,1]}{\operatorname{Var}} k(t, s)=\operatorname{Var}_{s \in[0, t]}(s(1-t))+\underset{s \in[t, 1]}{\operatorname{Var}}(t(1-s))=2 t(1-t) \leq \frac{1}{2}$. 
Then let $M_{1}+\frac{3}{2} M\|u\|=r$, hence $\|\mathbb{A} x\| \leq r, \mathbb{A}\left(B_{r}\right) \subseteq B_{r}$ is verified.

Step 2. $\mathbb{A}\left(B_{r}\right)$ is equiregulated.

For every $t_{0} \in[0,1)$, and for every $x \in B_{r}$, one has

$$
\begin{aligned}
\left|\mathbb{A} x(t)-\mathbb{A} x\left(t_{0}+\right)\right| & \mid \int_{0}^{t} s(1-t) f(s, x(s)) d s+\int_{t}^{1} t(1-s) f(s, x(s)) d s+\int_{0}^{t} s(1-t) g(s, x(s)) d u(s) \\
& +\int_{t}^{1} t(1-s) g(s, x(s)) d u(s)-\int_{0}^{t_{0}+} s\left(1-\left(t_{0}+\right)\right) f(s, x(s)) d s \\
& -\int_{t_{0}+}^{1}\left(t_{0}+\right)(1-s) f(s, x(s)) d s-\int_{0}^{t_{0}+} s\left(1-\left(t_{0}+\right)\right) g(s, x(s)) d u(s) \\
& -\int_{t_{0}+}^{1}\left(t_{0}+\right)(1-s) g(s, x(s)) d u(s) \mid \\
= & \mid \int_{t_{0}+}^{t}(s-t) f(s, x(s)) d s+\int_{0}^{t_{0}+} s\left(\left(t_{0}+\right)-t\right) f(s, x(s)) d s \\
& +\int_{t_{0}+}^{1}(1-s)\left(t-\left(t_{0}+\right)\right) f(s, x(s)) d s+\int_{t_{0}+}^{t}(s-t) g(s, x(s)) d u(s) \\
& +\int_{0}^{t_{0}+} s\left(\left(t_{0}+\right)-t\right) g(s, x(s)) d u(s)+\int_{t_{0}+}^{1}(1-s)\left(t-\left(t_{0}+\right)\right) g(s, x(s)) d u(s) \mid \\
\leq & \left|\int_{t_{0}+}^{t}(s-t) f(s, x(s)) d s\right|+\mid \int_{0}^{t_{0}+} s\left(\left(t_{0}+\right)-t\right) f(s, x(s)) d s \\
& +\int_{t_{0}+}^{1}(1-s)\left(t-\left(t_{0}+\right)\right) f(s, x(s)) d s|+| \int_{t_{0}+}^{t}(s-t) g(s, x(s)) d u(s) \mid \\
& +\left|\int_{0}^{t_{0}+} s\left(\left(t_{0}+\right)-t\right) g(s, x(s)) d u(s)+\int_{t_{0}+}^{1}(1-s)\left(t-\left(t_{0}+\right)\right) g(s, x(s)) d u(s)\right| \\
= & I_{1}+I_{2}+I_{3}+I_{4} .
\end{aligned}
$$

in which

$$
\begin{gathered}
I_{1}=\left|\int_{t_{0}+}^{t}(s-t) f(s, x(s)) d s\right| \\
I_{2}=\left|\int_{0}^{t_{0}+} s\left(\left(t_{0}+\right)-t\right) f(s, x(s)) d s+\int_{t_{0}+}^{1}(1-s)\left(t-\left(t_{0}+\right)\right) f(s, x(s)) d s\right|, \\
I_{3}=\left|\int_{t_{0}+}^{t}(s-t) g(s, x(s)) d u(s)\right|, \\
I_{4}=\left|\int_{0}^{t_{0}+} s\left(\left(t_{0}+\right)-t\right) g(s, x(s)) d u(s)+\int_{t_{0}+}^{1}(1-s)\left(t-\left(t_{0}+\right)\right) g(s, x(s)) d u(s)\right| .
\end{gathered}
$$

In what follows, we prove that $I_{1}, I_{2}, I_{3}$ and $I_{4}$ tend to 0 as $t$ tends to $t_{0}+$, respectively.

$$
\begin{aligned}
I_{2}= & \left|\int_{0}^{t_{0}+} s\left(\left(t_{0}+\right)-t\right) f(s, x(s)) d s+\int_{t_{0}+}^{1}(1-s)\left(t-\left(t_{0}+\right)\right) f(s, x(s)) d s\right| \\
\leq & \left|t-\left(t_{0}+\right)\right|\left(\left|\int_{0}^{t_{0}+} s f_{1}(s) d s\right|+\left|\int_{0}^{t_{0}+} s f_{2}(s) d s\right|+\left|\int_{t_{0}+}^{1}(1-s) f_{1}(s) d s\right|\right. \\
& \left.+\left|\int_{t_{0}+}^{1}(1-s) f_{2}(s) d s\right|\right) .
\end{aligned}
$$

In view of the assumption $\left(H_{3}\right)$, one knows that

$$
\left(\left|\int_{0}^{t_{0}+} s f_{1}(s) d s\right|+\left|\int_{0}^{t_{0}+} s f_{2}(s) d s\right|+\left|\int_{t_{0}+}^{1}(1-s) f_{1}(s) d s\right|+\left|\int_{t_{0}+}^{1}(1-s) f_{2}(s) d s\right|\right)
$$

is bounded. Therefore, $I_{2}$ tends to 0 as $t$ tends to $t_{0}+$. 
By the assumption $\left(H_{3}\right)$, we have

$$
I_{1}=\left|\int_{t_{0}+}^{t}(s-t) f(s, x(s)) d s\right| \leq\left|\int_{t_{0}+}^{t}(s-t) f_{1}(s) d s\right|+\left|\int_{t_{0}+}^{t}(s-t) f_{2}(s) d s\right| .
$$

Therefore, $I_{1}$ tends to 0 as $t$ tends to $t_{0}+$.

With the help of Lemma 3.3 and Lemma 3.4, we obtain

$$
\begin{aligned}
I_{4}= & \left|\int_{0}^{t_{0}+} s\left(\left(t_{0}+\right)-t\right) g(s, x(s)) d u(s)+\int_{t_{0}+}^{1}(1-s)\left(t-\left(t_{0}+\right)\right) g(s, x(s)) d u(s)\right| \\
= & \left|t-\left(t_{0}+\right)\right|\left(\left|\int_{0}^{t_{0}+} s g(s, x(s)) d u(s)+\int_{t_{0}+}^{1}(1-s) g(s, x(s)) d u(s)\right|\right) \\
\leq & \left|t-\left(t_{0}+\right)\right|\left(\underset{\left[0, t_{0}+\right]}{\operatorname{Var}}(s g(s, x(s)))+\left|\left(t_{0}+\right) g\left(\left(t_{0}+\right), x\left(t_{0}+\right)\right)\right|\right. \\
& \left.+\underset{\left[t_{0}+, 1\right]}{\operatorname{Var}}((1-s) g(s, x(s)))+\left|\left(1-\left(t_{0}+\right)\right) g\left(\left(t_{0}+\right), x\left(t_{0}+\right)\right)\right|\right)\|u\| \\
\leq & \left|t-\left(t_{0}+\right)\right|\left(\underset{\left[0, t_{0}+\right]}{\operatorname{Var}} s\|g(\cdot, x)\|+\underset{\left[0, t_{0}+\right]}{\operatorname{Var}} g(\cdot, x)\|s\|+M\right. \\
& \left.+\underset{\left[t_{0}+, 1\right]}{\operatorname{Var}}(1-s)\|g(\cdot, x)\|+\underset{\left[t_{0}+, 1\right]}{\operatorname{Var}} g(\cdot, x)\|1-s\|\right)\|u\| \\
\leq & 3 M\left|t-\left(t_{0}+\right)\right|\|u\| .
\end{aligned}
$$

Thus we know that $I_{4}$ tends to 0 as $t$ tends to $t_{0}+$.

$$
I_{3}=\left|\int_{t_{0}+}^{t}(s-t) g(s, x(s)) d u(s)\right| \leq\left|\int_{t_{0}+}^{t} s g(s, x(s)) d u(s)\right|+\left|t \int_{t_{0}+}^{t} g(s, x(s)) d u(s)\right| .
$$

According to the definition of the Henstock-Kurzweil-Stieltjes integral, we have

$$
\begin{aligned}
& \left|\int_{t_{0}+}^{t} g(s, x(s)) d u(s)\right| \\
& =\left|g(t, x(t)) u(t)-g\left(t_{0}+, x\left(t_{0}+\right)\right) u\left(t_{0}+\right)-\sum_{j=1}^{m}\left(g\left(\tau_{j}, x\left(\tau_{j}\right)\right)-g\left(\tau_{j-1}, x\left(\tau_{j-1}\right)\right)\right) u\left(t_{j-1}\right)\right| \\
& =\mid g(t, x(t))\left(u(t)-u\left(t_{0}+\right)\right)+\left(g(t, x(t))-g\left(t_{0}+, x\left(t_{0}+\right)\right)\right) u\left(t_{0}+\right)- \\
& \quad \sum_{j=1}^{m}\left(g\left(\tau_{j}, x\left(\tau_{j}\right)\right)-g\left(\tau_{j-1}, x\left(\tau_{j-1}\right)\right)\right) u\left(t_{j-1}\right) \mid \\
& =\mid g(t, x(t))\left(u(t)-u\left(t_{0}+\right)\right)+\left(\sum_{j=1}^{m}\left(g\left(\tau_{j}, x\left(\tau_{j}\right)\right)-g\left(\tau_{j-1}, x\left(\tau_{j-1}\right)\right)\right) u\left(t_{0}+\right)-\right. \\
& \quad \sum_{j=1}^{m}\left(g\left(\tau_{j}, x\left(\tau_{j}\right)\right)-g\left(\tau_{j-1}, x\left(\tau_{j-1}\right)\right)\right) u\left(t_{j-1}\right) \mid \\
& \leq\left|g(t, x(t))\left(u(t)-u\left(t_{0}+\right)\right)\right| \\
& \quad+\sum_{j=1}^{m}\left|g\left(\tau_{j}, x\left(\tau_{j}\right)\right)-g\left(\tau_{j-1}, x\left(\tau_{j-1}\right)\right)\right| \max _{s \in\left[t_{0}+, t\right]}\left|u\left(t_{0}+\right)-u(s)\right| \\
& \leq\left|g(t, x(t))\left(u(t)-u\left(t_{0}+\right)\right)\right|+2 M \max _{s \in\left[t_{0}+, t\right]}\left|u\left(t_{0}+\right)-u(s)\right| .
\end{aligned}
$$

Since $u$ is a regulated function, $u(t)-u\left(t_{0}+\right)$ tends to 0 as $t$ tends to $t_{0}+$. Thus we obtain that $\left|\int_{t_{0}+}^{t} g(s, x(s)) d u(s)\right|$ tends to 0 as $t$ tends to $t_{0}+$. 
Analogously, following the step above, we know that $\int_{t_{0}+}^{t} s g(s, x(s)) d u(s)$ tends to 0 as $t$ tends to $t_{0}+$. Therefore, $I_{3}$ tends to 0 as $t$ tends to $t_{0}+$.

For the reason that $I_{1}, I_{2}, I_{3}$ and $I_{4}$ tend to 0 as $t$ tends to $t_{0}+$, one obtains $\mid \mathbb{A} x(t)-$ $\mathbb{A} x\left(t_{0}+\right) \mid$ tends to 0 as $t$ tends to $t_{0}+$.

Similarly as done before, for every $t \in(0,1]$, and for every $x \in B_{r}$, we obtain that $\left|\mathbb{A} x(t)-\mathbb{A} x\left(t_{0}-\right)\right|$ tends to 0 as $t$ tends to $t_{0}-$.

So we have proved that $\mathbb{A}\left(B_{r}\right)$ is equiregulated.

Step 3. $S$ is bounded.

In view of Step 1, one has

$$
|x(t)|=|\lambda \mathbb{A} x(t)| \leq \lambda r, t \in[0,1] .
$$

Thus $S$ is bounded.

Step 4. A is continuous.

Considering a sequence $\left\{x_{n}\right\}_{n}(n=1,2,3, \ldots)$ converging uniformly to $x$ in $B_{r},\left(H_{2}\right)$ indicates that as $n \rightarrow \infty$,

$$
f\left(\cdot, x_{n}\right) \rightarrow f(\cdot, x), g\left(\cdot, x_{n}\right) \rightarrow g(\cdot, x) .
$$

As we know before, $B V[0,1]$ is a Banach space with the norm $\|\cdot\|_{B V}$, which yields $\lim _{n \rightarrow \infty}\left\|g_{n}-g\right\|_{B V}=0$. According to Lemma 3.5,

$$
\lim _{n \rightarrow \infty} \int_{0}^{1} k(t, s) g\left(s, x_{n}(s)\right) d u(s)=\int_{0}^{1} k(t, s) g(s, x(s)) d u(s) .
$$

And in virtue of assumption $\left(H_{3}\right)$ and Lemma 3.6,

$$
\lim _{n \rightarrow \infty} \int_{0}^{1} k(t, s) f\left(s, x_{n}(s)\right) d s=\int_{0}^{1} k(t, s) f(s, x(s)) d s .
$$

Then $\lim _{n \rightarrow \infty} \mathbb{A} x_{n}=\mathbb{A} x$. Consequently, $\mathbb{A}$ is continuous.

Combining all these steps above, we deduce that, according to Schaefer's fixed point theorem, $\mathbb{A}$ has at least one fixed point, which is the solution to problem (1.2) with the Dirichlet boundary condition (1.3).

Example 3.10. Consider the following Dirichlet problem

$$
\left\{\begin{array}{l}
-D^{2} x(t)=\sin (x(t))+H\left(t-\frac{2}{3}\right) D\left(W(t)+H\left(t-\frac{2}{3}\right)\right), \quad t \in[0,1] \\
x(0)=x(1)=0
\end{array}\right.
$$

in which $W(t)$ is the Weierstrass function

$$
W(t)=\sum_{n=1}^{\infty} \frac{\sin \left(3^{n} \pi t\right)}{2^{n}},
$$

and $H\left(t-\frac{2}{3}\right)$ is a Heaviside function. It is known that $W(t)$ is continuous but differentiable nowhere. Let $f(t, x)=\sin (x(t)), g(t, x)=H\left(t-\frac{2}{3}\right)$, and $u(t)=W(t)+H\left(t-\frac{2}{3}\right)$, for $t \in[0,1]$. One can verify that $f, g$ satisfy $\left(H_{1}\right)-\left(H_{4}\right)$. Thus Theorem 3.9 guarantees that problem (3.5) has at least one solution.

\section{Uniqueness of solution to problem (1.2) with the Dirichlet boundary condition (1.3)}

In this section, we study the uniqueness of solution to problem (1.2) with the Dirichlet boundary condition (1.3) by using the Banach's contraction mapping principle.

For the sake of introducing Banach's contraction mapping principle, we here give the definition of contraction mapping. 
Definition 4.1. Let $(M, d)$ be a compact metric space. A mapping $T: M \rightarrow M$ is said to be contraction mapping if there exists $0<\alpha<1$, such that

$$
d(T(x), T(y)) \leq \alpha d(x, y)
$$

for each $x, y \in M$.

Lemma $4.2([5])$. Let $(M, d)$ be a compact metric space and let $T: M \rightarrow M$ be a contraction mapping. Then $T$ has a unique fixed point in $M$.

The following assumption is necessary to our work.

$(H) f(\cdot, x(\cdot))$ is HK-integrable, $g(\cdot, x(\cdot)) \in B V[0,1]$, and for every $x, y \in G[0,1]$, there exist constants $L_{1}, L_{2}>0$ satisfying

$$
|f(t, x(t))-f(t, y(t))| \leq L_{1}|x(t)-y(t)|, \text { for every } x, y \in G([0,1], R), t \in[0,1] \text {; }
$$

$\operatorname{Var}_{s \in[0,1]}(k(t, s)(g(s, x(s))-g(s, y(s)))) \leq L_{2}\|x-y\|$, for every $x, y \in G[0,1], t \in[0,1]$.

Define an operator $\mathbb{A}$ as in Section 3, and one can easily verify that Lemma 3.7 holds under the assumption $(H)$.

Theorem 4.3. If $f, g$ satisfy the assumption $(H)$ and $L_{1}+L_{2}\|u\|<1$, then problem (1.2) with the Dirichlet boundary condition (1.3) has a unique solution in $G[0,1]$.

Proof. For every $x_{1}, x_{2} \in G[0,1]$, according to assumption $(H)$, one obtains for every $t \in[0,1]$

$$
k(t, s)|f(s, x(s))-f(s, y(s))| \leq L_{1} k(t, s)|x(s)-y(s)| \leq L_{1}\|x-y\| .
$$

We know from Lemma 3.7 that

$$
k(t, 0)=k(t, 1)=0 .
$$

Then, due to Lemma 3.3, we have

$$
\begin{aligned}
& \left|\mathbb{A} x_{1}(t)-\mathbb{A} x_{2}(t)\right| \\
= & \left|\int_{0}^{1} k(t, s)\left(f\left(s, x_{1}(s)\right)-f\left(s, x_{2}(s)\right)\right) d s+\int_{0}^{1} k(t, s)\left(g\left(s, x_{1}(s)\right)-g\left(s, x_{2}(s)\right)\right) d u(s)\right| \\
\leq & \int_{0}^{1} L_{1}\left\|x_{1}-x_{2}\right\| d s+\underset{s \in[0,1]}{\operatorname{Var}}\left(k(t, s)\left(g\left(s, x_{1}(s)\right)-g\left(s, x_{2}(s)\right)\right)\right)\|u\| \\
\leq & L_{1}\left\|x_{1}-x_{2}\right\|+L_{2}\left\|x_{1}-x_{2}\right\|\|u\| \\
= & \left(L_{1}+L_{2}\|u\|\right)\left\|x_{1}-x_{2}\right\| .
\end{aligned}
$$

Consequently, since $0<\left(L_{1}+L_{2}\|u\|\right)<1, \mathbb{A}$ is a contraction mapping, which means that $\mathbb{A}$ has just one fixed point. Hence the solution is determined uniquely.

Next we give an example which will apply Theorem 4.3.

Example 4.4. Consider the following Dirichlet problem,

$$
\left\{\begin{array}{l}
-D^{2} x(t)=\frac{1}{6} \sin (x(t))+x\left(\frac{1}{2}\right) D u(t), \quad t \in[0,1] \\
x(0)=x(1)=0
\end{array}\right.
$$

in which

$$
u(t)= \begin{cases}1, & t=0 \\ t \sin \left(\frac{\pi}{t}\right), & 0<t \leq 1\end{cases}
$$

Let $f(t, x)=\frac{1}{6} \sin (x(t)), g(t, x)=x\left(\frac{1}{2}\right), t \in[0,1]$. One can verify that $\|u\|=1$,

$$
\left|f\left(t, x_{1}(t)\right)-f\left(t, x_{2}(t)\right)\right|=\left|\frac{1}{6} \sin \left(x_{1}(t)\right)-\frac{1}{6} \sin \left(x_{2}(t)\right)\right| \leq \frac{1}{6}\left|x_{1}(t)-x_{2}(t)\right|,
$$




$$
\begin{aligned}
& \underset{[0,1]}{\operatorname{Var}}\left(k(t, s)\left(g\left(s, x_{1}\right)-g\left(s, x_{2}\right)\right)\right)=\underset{[0,1]}{\operatorname{Var}}\left(k(t, s)\left(x_{1}\left(\frac{1}{2}\right)-x_{2}\left(\frac{1}{2}\right)\right)\right) \\
& \quad=\underset{[0, t]}{\operatorname{Var}}\left(s(1-t)\left(x_{1}\left(\frac{1}{2}\right)-x_{2}\left(\frac{1}{2}\right)\right)\right)+\underset{[t, 1]}{\operatorname{Var}}\left(t(1-s)\left(x_{1}\left(\frac{1}{2}\right)-x_{2}\left(\frac{1}{2}\right)\right)\right) \\
& \quad \leq \frac{1}{2}\left|x_{1}\left(\frac{1}{2}\right)-x_{2}\left(\frac{1}{2}\right)\right| \\
& \quad \leq \frac{1}{2}\left\|x_{1}-x_{2}\right\| .
\end{aligned}
$$

Let $L_{1}=\frac{1}{6}, L_{2}=\frac{1}{2}$. It is easy to verify that $f$ and $g$ satisfy the assumption $(H)$ and $L_{1}+L_{2}\|u\| \leq \frac{2}{3}<1$. Consequently, Theorem 4.3 ensures a unique solution to problem (4.1).

Remark 4.5. In Example 3.10 and Example 4.4, $u$ is not a function of bounded variation but a regulated function. It implies that our theorems are nontrivial.

Acknowledgment. The authors are grateful to the referee for valuable comments and suggestions which are of great help to the improvement of the manuscript. This paper is supported by "the Fundamental Research Funds for the Central Universities"(No.:2017B19714, 2017B07414) and "Natural Science Foundation of Jiangsu Province"(No. BK20180500).

\section{References}

[1] J. Appell, J. Banaś and N. Merentes, Bounded variation and around, in: De Gruyter Series in Nonlinear Analysis and Applications, 17, De Gruyter, Berlin, 2014.

[2] P.C. Das and R.R. Sharma, Existence and stability of measure differential equations, Czechoslovak Math. J. 22 (97), 145-158, 1972.

[3] M. Federson, R. Grau, J.G. Mesquita and E. Toon, Boundedness of solutions of measure differential equations and dynamic equations on time scales, J. Differential Equations, 263 (1), 26-56, 2017.

[4] D. Fraňková, Regulated functions, Math. Bohem. 116 (1), 20-59, 1991.

[5] W.A. Kirk and B. Sims, Handbook of Metric Fixed Point Theory, Kluwer Academic Publishers, Dordrecht, 2001.

[6] P.Y. Lee, Lanzhou lectures on Henstock integration, World Scientific, Singapore, 1989.

[7] S. Leela, Stability of measure differential equations, Pacific J. Math. 55, 489-498, 1974.

[8] G.A. Monteiro and A. Slavík, Linear measure functional differential equations with infinite delay, Math. Nachr. 287 (11-12), 1363-1382, 2014.

[9] G.A. Monteiro and A. Slavík, Extremal solutions of measure differential equations, J. Math. Anal. Appl. 444 (1), 568-597, 2016.

[10] A. Slavík, Measure functional differential equations with infinite delay, Nonlinear Anal. 79, 140-155, 2013.

[11] A. Slavík, Well-posedness results for abstract generalized differential equations and measure functional differential equations, J. Differential Equations, 259 (2), 666-707, 2015.

[12] M. Tvrdý, Differential and integral equations in the space of regulated functions, Mem. Differential Equations Math. Phys. 25, 1-104, 2002.

[13] G. Ye and W. Liu, The distributional Henstock-Kurzweil integral and applications, Monatsh. Math. 181 (4), 975-989, 2016.

[14] J.H. Yoon, G.S. Eun and Y.C. Lee, On Henstock-Stieltjes integral, Kangweon Kyungki J. Math. 6, 87-96, 1998.

[15] E. Zeidler, Nonlinear Functional Analysis and its Applications, I - Fixed-Point Theorems, Springer-Verlag, New York, 1986. 